\title{
Acute Presentation and Management of Abdominal Tuberculosis
}

\author{
Qamar Ashfaq Ahmad, Muhammad Zeeshan Sarwar, Nafeesah Fatimah, Abubaker Shafiq Ahmed, \\ Shabbar Hussain Changaizi and Mahmood Ayyaz \\ Department of General Surgery, Services Hospital, Lahore, Pakistan
}

\begin{abstract}
Objective: To study the clinicopatholgical profile and outcomes of surgical management of abdominal tuberculosis (ATB) Study Design: Cross-sectional observational study.

Place and Duration of Study: Department of Surgery, Services Hospital, Lahore, Pakistan, from May 2008 to April 2018. Methodology: All patients who underwent emergency laparotomy during the study period due to abdominal tuberculosis, and consented to participate in the study were included. Demographic variables and type of surgical procedure performed were recorded. Patients were followed-up for histopathology, recurrence, or any anti-tuberculous therapy related complications at $1,4,6$, and 12 months. Data were analysed using SPSS version 21.

Results: Out of the 80 patients, 36 were males and 44 were females. The median age was 23.5 years (range $=11-90$ years). Mean weight of the patients was $48.7 \pm 12.2 \mathrm{~kg}$. Commonest presenting symptom was abdominal pain $72(90 \%)$. On exploration, ileocecal region was most commonly involved segment 68 (85\%). Stoma formation was the most common surgical procedure performed in $59(73.8 \%)$ patients. Complications and mortality rate were $48(60 \%)$ and $7(8.7 \%)$, respectively. A significant relationship of complications was found with prolonged hospital stay $(p<0.001)$.

Conclusion: Abdominal tuberculosis is a major public health concern. Vague symptoms lead to diagnostic delay so patients present late with intestinal obstruction. lleocecal tuberculosis is the most common site of involvement.
\end{abstract}

Key Words: Intestinal obstruction, Abdominal tuberculosis, Acute abdomen.

How to cite this article: Ahmad QA, Sarwar MZ, Fatimah N, Ahmed AS, Changaizi SH, Ayyaz M. Acute presentation and management of abdominal tuberculosis. J Coll Physicians Surg Pak 2020; 30(2):129-133.

\section{INTRODUCTION}

Tuberculosis (TB) is one of the top ten causes of death worldwide. In 2016, 10.4 million people fell ill with TB and 1.7 million died from the disease. Over $95 \%$ of the deaths occur in low- and middle-income countries. Pakistan is among the top seven countries sharing $64 \%$ burden of worldwide tuberculosis, producing 510,000 new cases annually.1,2 Abdominal tuberculosis (ATB) is the sixth most prevalent presentation of extra-pulmonary disease, accounting for one in five registered TB patients. ${ }^{3}$ In Pakistan, ATB remains the most common extra-pulmonary site.

Abdominal tuberculosis involves gastrointestinal tract, peritoneum, lymph nodes and solid viscera (liver, spleen and pancreas).4,5,8 Intestinal tuberculosis exists in various forms, i.e., ulcerative, hypertrophic or ulcerohypertrophic and fibrous (stricture) type, while peritoneal tuberculosis may present as various forms such as ascitic, loculated, plastic, and purulent forms. Mesenteric and retro-peritoneal lymph nodes may get caseated and calcify later. ${ }^{5}$ Focal granulomas may be found involving

Correspondence to: Dr. Nafeesah Fatimah, Department of General Surgery, Services Hospital, Lahore, Pakistan

E-mail:nafeesahjavaid@gmail.com

Received: September 30, 2019; Revised: December 30, 2019;

Accepted: January 24, 2020 the solid intra-abdominal viscera. Clinically, it may present in acute, chronic or acute on chronic form. Commonly, it runs a chronic course with non-specific symptoms of fever $(40-70 \%)$, pain $(80-95 \%)$, diarrhea $(11-20 \%)$, constipation, alternating constipation and diarrhea, weight loss (40-90\%), anorexia and malaise.4-7

The diagnosis of abdominal tuberculosis is considered difficult because symptoms are diverse and vague with lack of specific diagnostic testing. 3-5 Abdominal symptoms mimic other gastrointestinal diseases such as inflammatory bowel diseases, intestinal lymphomas or malignancy. 8 This dilemma results in delays in diagnosis and treatment, leading to significant morbidity and mortality. Hence, most of the cases of abdominal tuberculosis present in surgical emergency with obstruction and peritonitis. In such situations, surgery plays a pivotal role in the diagnosis and treatment.

Due to lack of consensus guidelines, surgical management of ATB remains a challenge for surgeons. Bypass surgery, segmental resection (one-stage or two-stage) and stricturoplasty are among the most frequently documented surgical procedures. ${ }^{7-10}$ Various factors affecting the decision are site and extent of the disease, status of the remaining gut, general condition of the patient, expertise available in emergency, preference of the individual surgeon and institutional protocols. As this disease is prevalent is South Asia, good quality obser- 
vational data from Pakistan and neighbouring countries is required for formulation of these guidelines.

The aim of this study was to determine clinical and radiological features of abdominal tuberculosis and their surgical management options.

\section{METHODOLOGY}

An observational study was done in Department of Surgery, Services Hospital, Lahore from May 2008 to April 2018. Ethical review was sought from Institutional Review Board, Services Hospital, Lahore. Records of the patients who underwent emergency laparotomy with intestinal obstruction and peritonitis, and whose diagnosis later on confirmed on histopathology to be tuberculosis were included in the study. Patients presenting with perforations other than abdominal/ peritoneal tuberculosis were excluded.

Detailed case history, physical examination and relevant laboratory examination were evaluated. All patients were optimised with intravenous hydration, nasogastric intubation and Foley's catheterisation where needed. After adequate resuscitation, laparotomy under general anaesthesia with endotracheal intubation was done through midline incision. Postoperatively, patients were admitted in surgical ward and started on anti-tuberculous regimen including intramuscular streptomycin for first three months. Patients were followed-up one week after discharge and then at 1, 4, 6, and 12 months time.

Data were analysed using Statistical Package for Social Sciences 21.0 for Windows (SPSS Inc., Chicago, Illinois, United States of America). Numerical data such as age, weight, hospital stay, were expressed as mean \pm standard deviation. Categorical data such as gender, symptoms, presentation, investigation, operative findings, procedure and postoperative complications were expressed as frequencies and proportions. T-test was applied to compare the two means. A $p<0.05$ was considered statistically significant.

\section{RESULTS}

Eighty patients were enrolled in the study. The sample had $36(45 \%)$ males and $44(55 \%)$ females with male to female ratio of 1:1.2. The age of the patients ranged from 11 to 90 years with a mean age of $26.5 \pm 13.4$ (Interquartile range IQR $=17.3$ to 30.0 ) years (Figure 1). Mean weight of the population was $48.7 \pm 12.2 \mathrm{kgs}$. Thirty-three (41\%) patients had body mass index (BMI) $<18 \mathrm{~kg} / \mathrm{m}^{2}$. Majority of the patients, $56(70 \%)$, had no formal education and $40(50 \%)$ were unemployed. Most of our sample, $66(83 \%)$, belonged to rural areas and 63 $(79 \%)$ were from poor socioeconomic status.

The majority of the patients, 59 (73.8\%) had symptoms for more than 6 months duration at the time of presentation. The commonest presenting symptom was abdominal pain $72(90 \%)$, followed by vomiting and constipation in $62(78 \%)$ patients (Table I). A total of 16 $(20 \%)$ patients had prior history of treatment for tuberculosis, $13(16.3 \%)$ had positive family history of tuberculosis, while $10(12.5 \%)$ patients had concomitant pulmonary tuberculosis.

On baseline investigations, the hemoglobin level was found to be less than $10 \mathrm{~g} / \mathrm{dl}$ in $59(73.7 \%)$ patients. Electrolyte abnormalities were seen in 50 (62.5\%) patients. Plain radiography of the abdomen revealed multiple dilated small bowel loops with significant air fluid levels in $48(60.0 \%)$ patients. Free air under right hemidiaphragm were seen in $21(26.2 \%)$ patients. Abdominal ultrasound and computed tomography (CT) imaging was done in $55(68.7 \%)$ and $19(23.7 \%)$ patients, respectively with majority demonstrating dilated gut loops with airfluid levels. Omental thickening $32(40 \%)$, mesenteric fat stranding $28(35 \%)$, and mesenteric lymph node enlargement 10 (13\%) were seen on CT imagining.

On exploration, small intestine was involved with tuberculosis in 75 (93.8\%) patients, peritoneum was involved in $3(3.6 \%)$ patients and mesenteric nodes were involved in $2(2.5 \%)$ patients, while one patient had pelvic tuberculosis involving the fallopian tubes. The most commonly involved bowel segment was ileum and ileocecal region in $68(85.0 \%)$ patients followed by jejunum in $7(9.3 \%)$ patients. Intestinal resection and covering ileostomy was the most frequent surgical procedure performed in 59 $(73.8 \%)$ patients (Table II). Forty-eight complications

Table I: Distribution of patients according to clinical presentation.

\begin{tabular}{ll}
\hline Symptoms & No. (\%) \\
\hline Abdominal Pain & $72(90)$ \\
Vomiting & $62(78)$ \\
Constipation & $62(78)$ \\
Weight loss & $46(58)$ \\
Peritonism & $43(54)$ \\
Abdominal distention & $29(36)$ \\
Constipation & $29(36)$ \\
Abdominal mass & $19(24)$ \\
\hline
\end{tabular}

Table II: Distribution of patients according to operative procedures and complications.

\begin{tabular}{lc}
\hline Operative procedure & $59(73.8 \%)$ \\
Stoma & $11(13.7 \%)$ \\
Primary anastomosis & $4(5.00 \%)$ \\
Stricturoplasty & $6(7.50 \%)$ \\
Biopsy only & \\
\hline Complications: & $21(43.7 \%)$ \\
Wound Infection & $4(8.3 \%)$ \\
Wound dehiscence & $3(6.25 \%)$ \\
Abdominal collection & $5(10.4 \%)$ \\
ATT complications & $5(10.4 \%)$ \\
Stoma complications & $4(8.3 \%)$ \\
Enterocutaneous fistula & $2(4.2 \%)$ \\
Chest Infection & $4(8.3 \%)$ \\
Septicemia, MODs & \\
\hline
\end{tabular}




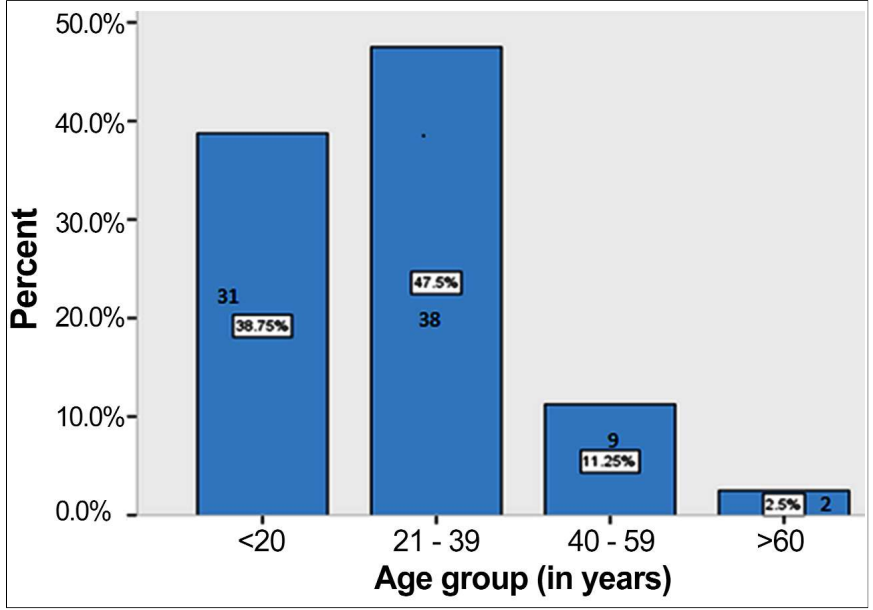

Figure 1: Age distribution of cases as per age group.

The numbers in the bars represent the total number of cases falling in the certain age group.

were recorded, of which surgical site infection was the most common complication accounting for $43.8 \%$ (35) of cases (Table II). A significant relationship of complications was found with prolonged hospital stay $(p<0.001$, Table III). Histological examination of the biopsy specimen was performed in all cases and revealed chronic granulomatous inflammation with caseating necrosis.

The overall length of hospital stay was quite variable and ranged from 2 to 90 days with mean stay of $14.9 \pm 11.5$ days. Patients with stoma formation were more likely to have prolonged hospital stay, i.e. more than seven days. Antituberculous therapy was started. For the first three months, treatment regimen involved Inj. streptomycin in addition to routine first line therapy (isoniazid, rifampicin, ethambutol and pyrazinamide). Rest of the six months were competed by two drugs. Almost whole population (95\%) completed antituberculous therapy for 9 months. However, five patients developed complications of ATT, which was settled with modification of therapy. Sixty-nine $(86 \%)$ patients were available for follow-up at 12 months after discharge. None of the patients had relapse of disease during this follow-up period. Out of the 80 patients, 7 (8.8\%) patients expired during hospital stay.

\section{DISCUSSION}

Tuberculosis has remained a source of significant morbidity and mortality all over the world with developing countries being on the lead; among them, Pakistan has remained in the top ten.1,2 Not only pulmonary tuberculosis but abdominal tuberculosis has also been widely prevalent in Pakistan because of poor sanitation, overcrowding, poverty, and lack of access to healthcare facilities.1-3 Abdominal tuberculosis has particularly remained a diagnostic challenge because of varied presentations. Moreover, being a disease prevalent in poor households and in areas where access to healthcare facilities is limited, abdominal tuberculosis usually presents late, mostly in surgical emergencies with obstruction and peritonitis. This study was conducted on eighty cases presenting in surgical emergency.

The mean age was found to be 23.5 years with the youngest patient being eleven years old and the oldest being 90 years old. This finding was consistent with other studies that have reported peak incidence of tuberculosis in $2^{\text {nd }}$ to $4^{\text {th }}$ decades.3,7 However, in the developed world, abdominal tuberculosis is mostly seen in older, immunocompromised patients or in immigrants from endemic areas. ${ }^{15,19}$ The peak incidence of abdominal tuberculosis in the productive years of life puts a great impact on the economy of the family and the society as a whole.

The slight female preponderance (44 vs. 36) was noted in this study. The gender difference have remained variable. Some authors have reported predominance of the disease in females in developing countries, while some studies show male predominance. ${ }^{8-11}$ However, no significant reason could be found for this gender difference.

A total of $33 / 80(41 \%)$ of the population in our study was in the underweight category as per WHO BMI classification. This finding was consistent to other studies, which have also highlighted the fact that abdominal tuberculosis was mostly seen in under-nourished patients. 9,12

In this study, majority of patients presented with more than 24-48 hours duration of intestinal obstruction and/or peritonitis, as noted in other studies. $13,14,18$ Malnourishment, overcrowding, and illiteracy stemming from poverty are the major factors leading to delay in seeking healthcare as seen in this data, i.e. $70 \%$ of our patients had no formal education and $50 \%$ of our patients were unemployed. Similar pattern was seen in the literature.2,13 Moreover, there are other sociocultural causes of the delay, i.e. visiting quacks and seeking advice from spiritual healers, which is attributable to low literacy and lack of awareness of the disease. Public health consultants and health policy-makers need to organise public awareness campaigns focusing illiterate population for early diagnosis and prompt management of the disease.

Only ten patients $(12 \%)$ had a concomitant pulmonary tuberculosis, while majority had primary abdominal tuberculosis. Various studies done in developing countries have shown similar results while in developed countries, primary tuberculosis is rare. However, no logical explanation could be drawn for such differences. ${ }^{3,14}$

In agreement with the other studies, ileocecal region was the most commonly involved region in this study $(90.7 \%) .6,12,14,15$ Similar figures have been provided in previous studies studying acute presentations of abdominal tuberculosis with ileal strictures being the most prevalent pathology. 5,12 This could be explained by the abundance of Peyer's patches in the ileal segment, 
which lead to uptake of mycobacterium tuberculosis by lymphoid cells in this region and proliferation of lymphoid tissue. Moreover, stasis and increased physiological exchange of fluid and electrolytes lead to increase in the contact time of pathogens with ileal mucosa rendering this region vulnerable for the development of intestinal tuberculosis. ${ }^{16-20}$

Diagnosis was made depending on histopathology in our study. Routine microscopy with AFB stain, tissue culture, and PCR can be used for diagnosis. ${ }^{16}$ In this study, no record of above investigations could be found as the tissue culture and PCR for mycobacterium tuberculosis is done in specialised centres only and was not available at the study centre.

Most commonly performed procedure was resection of the diseased segment and ileostomy. Although no statistically significant difference was noted in patients who underwent stoma formation compared to those who underwent primary anastomosis, comparison cannot be properly drawn because no randomisation was observed in selecting patients for either primary anastomosis or stoma formation. Stoma needs proper patient education for handling and prolongs hospital stay. ${ }^{17,18}$ Moreover, second surgery is required to reestablish intestinal continuity once the disease is cured, increasing significantly the morbidity. Hence, randomised controlled trials are needed to establish whether primary anastomosis is superior to stoma in the management of intestinal tuberculosis presenting as acute abdomen. Nonetheless, randomisation is particularly a difficult task in such a study because of varied presentations of abdominal tuberculosis. Moreover, experience has shown that stoma prevents significant morbidity in moribund patients presenting with more than 48 hours of peritonitis; and intra-abdominal sepsis increases the chances of anastomotic breakdown; however, evidencebase is lacking in such cases. ${ }^{20-22}$

Most common surgical complication in this study was surgical site infection (SSI) (43.8\%). This incidence was similar to that observed in other studies, which also had SSI as major complication. 3,16,17 However, a few reported respiratory complications as the most predominant. $5 \mathrm{SSI}$ was observed in this study because most of our patients were undernourished and, hence, had reduced healing ability in addition to decreased immune function.

All the patients were given anti-tuberculous (ATT) regimen for 9-12 months. In that duration, they were monitored for any ATT-induced complications, which were seen in five patients. Despite its limitations, this study throws some light on the spectrum of a highly prevalent disease in South Asia. Being common in illiterate, low socioeconomic groups, it poses a significant challenge to public health specialists, health-policy makers, and infectious disease experts. The local data can be used to design strategies for prevention and early identification of the disease. Late presentation, inadequate drug treatment, poor follow-up has led to the emergence of drug-resistant strains. These problems need to be addressed to deliver appropriate care to our patients.

\section{CONCLUSION}

Abdominal tuberculosis is a major public health concern of low-income countries. It remains a diagnostic dilemma for healthcare providers because of diverse presentations and unequivocal signs and symptoms. It is seen in undernourished people of relatively younger age group. Delayed presentation remains the hallmark of this disease in our setting. Hence, disease surveillance strate-gies need to be implemented to ascertain early diagnosis and prompt treatment. Moreover, more randomised controlled-trials need to be conducted to establish evidence-based guidelines on the surgical management of acute abdomen due to abdominal tuberculosis.

\section{ETHICAL APPROVAL:}

Ethical review was sought from Institutional Review Board, Services Hospital, Lahore.

\section{PATIENTS' CONSENT:}

Written informed consents were sought from patients before enrolling them in the study.

\section{CONFLICT OF INTEREST:}

Authors declared no conflict of interest.

\section{AUTHORS' CONTRIBUTION:}

QAA: Designed the study, collected and analysed the data, and was involved in manuscript writing.

MZS: Designed the study, was involved in data analysis, and manuscript writing.

NF: Involved in formulation of study design, data collection, data analysis, manuscript writing, and final approval of the manuscript

ASA: Collected and analysed the data and helped in manuscript writing.

SHC: Helped in data analysis, manuscript writing, and approval of final version

MA: Supervised all the works and every step of this study from formulation of study question to the final approval of the manuscript.

\section{REFERENCES}

1. Resource Center, National TB Control Program. https://www. ntp.gov.pk/resource.php, 2018 [Accessed on 20 May 2018].

2. Tuberculosis in Pakistan, World Health Organization Eastern Mediterranean Region. http://www.emro.who.int/pak/programmes/ stop-tuberculosis.html, 2018 [Accessed on 20 May 2018].

3. Shaikh R, Ayub M, Malik KA, Jaleel M. Abdominal tuberculosis: Profile of 26 cases. Pakistan J Surg 2008; 24:217-9.

4. Chalya PL, Mchembe MD, Mshana SE, Rambau PF, Jaka H, Mabula JB. Clinicopathological profile and surgical treatment 
of abdominal tuberculosis: A single centre experience in northwestern tanzania. BMC Infect Dis 2013; 13:270.

5. Shaikh MS, Dholia KR, Jalbani MA, Shaikh SA. Prevalence of intestinal tuberculosis in cases of acute abdomen. Pakistan $J$ Surg 2007; 23:52-6.

6. Engin G, Balk E. Imaging findings of intestinal tuberculosis. J Comput Assist Tomogr 2005; 29:37-41.

7. Wani MU, Parvez M, Kumar SH, Naikoo GM, Jan M, Wani HA. Study of surgical emergencies of tubercular abdomen in developing countries. Indian J Surg 2015; 77:182-5.

8. Shukla S, Kumar K. Spectrum of clinical presentation of abdominal tuberculosis and its surgical management. Int Surg J 2018; 5: 1482-7.

9. Farmaullah I, Mumtaz K. Surgical management of abdominal tuberculosis. J Postgrad Med Inst 2003; 17:33-41.

10. Bali RS, Jain R, Zahoor Y, Mittal A. Abdominal tuberculosis: A surgical emergency. Int J Res Med Sci 2017; 5:3847-50.

11. Baloch NA, Baloch MA, Baloch FA. A study of 86 cases of abdominal tuberculosis. J Surg Pak 2008; 13:30-2.

12. Ali WM, Ahmed M, Rab AZ, Rizvi SAA, Rafiq R, Rabbani SS. Acute presentation and management of abdominal tuberculosis at a tertiary care centre. J Evid Based Med Healthc 2017; 4:1937-41.

13. Mukhopadhyay A, Dey R, Bhattacharya U. Adbominal tuberculosis with an acute abdomen: Our experience. J Clin Diag Res 2014; 8:NC07-9.
14. Charokar K, Garg N, Jain KA. Surgical management of abdominal tuberculosis: A retrospective study from Central India. Int Surg J 2016; 3:23-31.

15. Kumar S, Pandey HI, Saggu P. Abdominal tuberculosis. In: Taylor I, Johnson Cd (Eds). Recent Adv Surg 2008; 28:47-58.

16. Johar R, Chawla K, Mukhopadhyay $C$. Is PCR better than culture in TB diagnosis: Myth or reality? BMC Infect Dis 2014; 14(Suppl3):46.

17. Abro A, Siddiqui FG, Akhtar S, Memon AS. Spectrum of clinical presentation and surgical management of intestinal tuberculosis at a tertiary care hospital. J Ayub Med Coll Abbottabad 2010; 22:96-9.

18. Urabinahatti AK, Singh AK, Nayak A, Gupta R, Jain M, Dubey C, et al. Abdominal tuberculosis: An epidemiological profile and management of 40 cases in a tertiary set up. Int Surg J 2016; 3:1502-8.

19. Hiremath SV, Nadaf SC. Surgical approach to abdominal tuberculosis. J Evid Based Med Healthc 2017; 4:3735-8.

20. Giannakopoulos GF, Veenhof A, Van Der Peet DL, Sietses C, Meijerink WJHJ, Cuesta MA. Morbidity and complications of protective loop ileostomy. Colorectal Dis 2009; 11:609-12.

21. Debi U, Ravisankar V, Prasad KK, Sinha SK, Sharma AK. Abdominal tuberculosis of the gastrointestinal tract: Revisited. World J Gastroenterol 2014; 20:14831-40.

22. Weledji PE, Pokem TB. Abdominal tuberculosis: Is there a role for surgery? World J Gastrointest Surg 2017; 9:174-81. 